\title{
Lattice Parameters of Aluminium Nitride in the Range 10-291 K
}

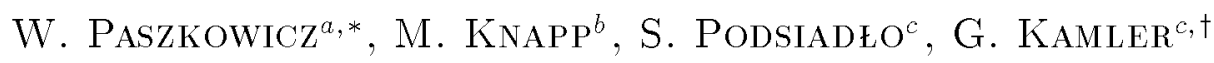 \\ AND J.B. PEŁKA ${ }^{a}$ \\ ${ }^{a}$ Institute of Physics, Polish Academy of Sciences \\ al. Lotników 32/46, 02-668 Warsaw, Poland \\ ${ }^{b}$ Darmstadt University of Technology, Institute of Materials Science \\ Petersenstr. 23, 64287 Darmstadt, Germany \\ ${ }^{c}$ Faculty of Chemistry, Warsaw University of Technology \\ Noakowskiego 3, 00-664 Warsaw, Poland

\begin{abstract}
Lattice parameters for aluminium nitride were determined using X-ray powder diffraction at a synchrotron radiation source (beamline B2, Hasylab/DESY, Hamburg) in the temperature range from $10 \mathrm{~K}$ to $291 \mathrm{~K}$. The measurements were carried out using the Debye-Scherrer geometry. The relative change of both, $a$ and $c$, on rising the temperature in the studied range $(10-291 \mathrm{~K})$ is about $0.03 \%$. The results are compared with earlier laboratory data and theoretical predictions.
\end{abstract}

PACS numbers: 81.05.Je, 65.40.De, 65.60.+a, 65.40.-b

\section{Introduction}

Aluminium nitride, AlN (wurtzite structure type) has specific physical properties like high thermal conductivity, high melting point, and large energy gap. Its applications as a component of refractory ceramics or buffer layers for GaN epilayers grown on sapphire are widely known. Elastic properties of each component of such layered structures and devices are an important factor influencing the properties of the product. Therefore, detailed studies of lattice parameter as a function of temperature may be helpful in design and improving the technologies involving aluminium nitride.

\footnotetext{
*corresponding author; e-mail: paszk@ifpan.edu.pl

${ }^{\dagger}$ present address: High-Pressure Research Center, Polish Academy of Sciences, Sokołowska 29/37, 01-142 Warsaw, Poland
} 
Early work on (mainly high-temperature) thermal expansion of AlN has been briefly summarized in $[1,2]$. Recent studies include experimental works on low-temperature [3] and high-temperature [4] expansivity, and calculations based on semi-empirical models [5]. As many other materials built from covalently bonded light atoms, AlN is characterized by low thermal expansivity. The experiments [3] and calculations in [5] indicate an existence of a domain of slightly negative expansion below about $100 \mathrm{~K}$ for both lattice parameters, the highest absolute value being of the order of $10^{-7} \mathrm{~K}^{-1}$. According to Refs. [3, 5], the variation of lattice parameters below $200 \mathrm{~K}$ is as low as about $0.0002 \AA$. In the present paper, the results of experimental investigation of the unit-cell dimensions for AlN in the temperature range $10-291 \mathrm{~K}$ are presented.

\section{Experimental}

Fine AlN powder was synthesized at Warsaw University of Technology from aluminium and urea in the reaction in ammonia atmosphere using the method described in $[6,7]$. Fine aluminium powder (grain size $0.5 \mu \mathrm{m}$ ) and urea were milled in the mortar under nitrogen atmosphere. The obtained mixture was heated in the tube furnace in the stream of ammonia in three steps: $0.5 \mathrm{~h}$ at $185^{\circ} \mathrm{C}, 0.5 \mathrm{~h}$ at $250^{\circ} \mathrm{C}$ and $3 \mathrm{~h}$ at $1400^{\circ} \mathrm{C}$. The prepared powder was stored in a closed container in order to protect it against interaction with air. The final AIN product contains less than $0.08 \%$ carbon. The size of the grains is $\approx 2 \mu \mathrm{m}[6]$.

The measurements were carried out at a high-resolution powder diffractometer at the B2 beamline using Debye-Scherrer geometry. The experiment consisted in data collection using temperature steps starting from the lowest available temperature, $10 \mathrm{~K}$. An NaI:Tl scintillation counter was applied for the data collection. The wavelength was determined to be $\lambda=1.20720(5) \AA$ by least squares refinement of five reflections of silicon powder (NIST 640b diffraction standard, $a=5.43094 \AA$ ). The AlN powder was mounted within a thin-wall capillary of $25 \mathrm{~mm}$ length, $1 \mathrm{~mm}$ diameter and $0.01 \mathrm{~mm}$ wall thickness. The diffraction experiments were carried out with the use of a He-closed-cycle cryostat (CTI model 21 SC Cryophysics) at a low helium pressure ensuring absence of thermal gradients and allowing capillary rotation (described in Ref. [8]). The temperature was kept constant within $1 \mathrm{~K}$ using a PID controller and a silicon diode temperature sensor.

Powder diffraction patterns were measured with a powder diffractometer installed at the B2 beamline at Hasylab/DESY, Hamburg, using a parallel beam monochromatized with a $\mathrm{Ge}(111)$ double crystal monochromator. Improved resolution of the diffractometer is ensured by a set of parallel horizontal foils at the diffracted beam of angular aperture $0.23^{\circ}$. The wavelength was determined to be $1.20720 \AA$ by a least squares procedure for a silicon NBS 640 b standard (with the lattice parameter $a_{\mathrm{Si}}=5.43094 \AA$ ). The data were collected at selected angular intervals ranging up to $98^{\circ}(2 \theta)$. A Rietveld refinement program, DBWS v. 94.11 [9] was used for evaluation of the lattice parameters. 


\section{Results and discussion}

Phase analysis revealed the presence of a small amount of aluminium oxide impurity (lines 012, 104, 113, 116 identified on the basis of Ref. [10], all of relative peak-height intensity as low as about $0.5 \%$ ). The lattice-parameter dependence on temperature is shown in Fig. 1. Polynomial fitting yields the following relations:

$$
\begin{aligned}
& a=3.11054+6.19 \times 10^{-9} T^{2}+5.82 \times 10^{-12} T^{3}, \\
& c=4.98006+2.82 \times 10^{-8} T^{2}+1.35 \times 10^{-10} T^{3} .
\end{aligned}
$$
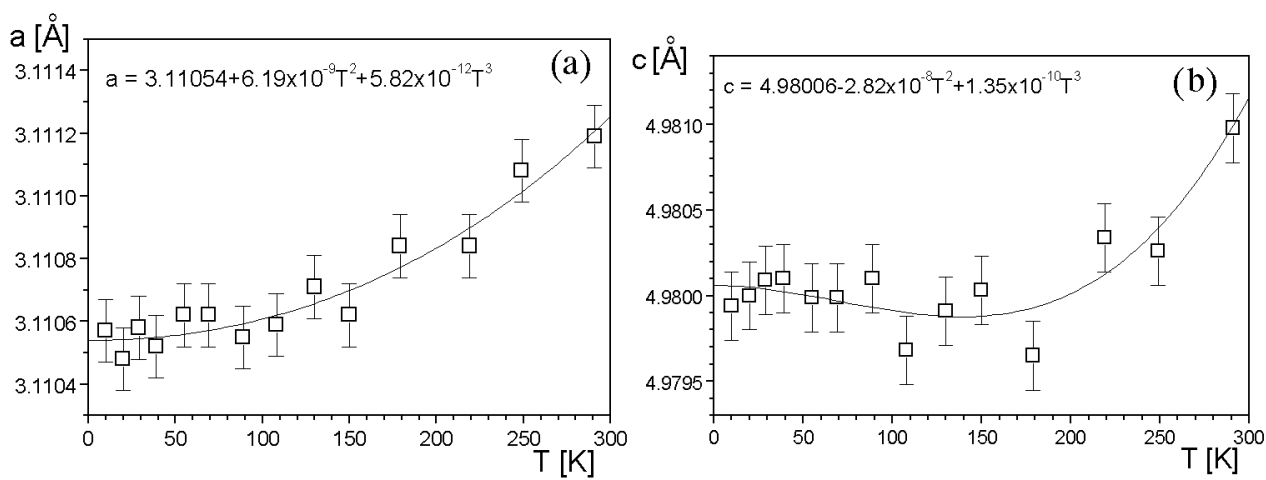

Fig. 1. Dependence of lattice parameters, $a$ and $c$, on temperature for aluminium nitride (empty squares). The solid lines represent polynomial fits of the experimental data.

Measured room-temperature $(291 \mathrm{~K})$ values are $a_{\mathrm{RT}}=3.1112(1) \AA, c_{\mathrm{RT}}=$ $4.9810(2) \AA$. Rietveld refinement of a full pattern collected at a Bragg-Brentano diffractometer yielded similar values, $a_{\mathrm{RT}}=3.1115(1) \AA, c_{\mathrm{RT}}=4.9810(2) \AA$ [11]. The relative increase in both parameters, $a$ and $c$, on rising the temperature in the studied range $(10-291 \mathrm{~K})$ is about $0.03 \%$. The axial ratio at $T=10 \mathrm{~K}$ is 1.6010 and its relative changes with increasing temperature are small. A scatter of $a_{\mathrm{RT}}$ and $c_{\mathrm{RT}}$ values quoted in literature for single and polycrystals synthesized by various methods is of the order of $0.1 \%$ (see Table) and in this sense the present results are consistent with other data. The dependence of both lattice parameters on temperature is shown in Fig. 1. Below about $150 \mathrm{~K}$ they are almost constant (their changes are comparable with the experimental errors of about $0.0001 \AA$ for $a$ and $0.0002 \AA$ for $c$ ). Although the runs do not indicate any minimum for $a$ and a possible shallow minimum for $c$, the magnitude of experimental errors does not allow for confirming the existence/absence of negative thermal expansion (predicted in [5] for both, $a$ and $c$, variables) without additional experimental effort. 
TABLE

Reported (chronologically ordered) room-temperature (R.T.) lattice parameters and axial ratio for AIN. The following radiation was applied in the reported studies: $\mathrm{Cr} K_{\alpha}$ [3], $\mathrm{Cu} K_{a}[11,13-16]$, synchrotron radiation (this work).

\begin{tabular}{l|l|l|c|l|c}
\hline \hline \multicolumn{1}{c|}{$a_{\mathrm{RT}}[\AA]$} & \multicolumn{1}{|c|}{$c_{\mathrm{RT}}[\AA]$} & \multicolumn{1}{|c|}{$c / a$} & $T[\mathrm{~K}]$ & \multicolumn{1}{|c|}{ Experimental details } & References \\
\hline 3.1129 & 4.9819 & 1.6004 & 298 & & {$[12]$} \\
$3.1105(5)$ & $4.9788(8)$ & $1.6006(5)$ & 297 & oxygen impurity <1\% & {$[1]$} \\
3.1115 & 4.9798 & 1.6004 & 291 & & {$[13]$} \\
$3.1114(1)$ & $4.9792(2)$ & 1.6003 & 298 & & {$[14]$} \\
3.1106 & 4.9795 & 1.6008 & R.T. & single crystal, oxygen impu- & {$[15]$} \\
& & & & rity 800 ppm & \\
3.1111 & 4.9751 & 1.5991 & 300 & $\begin{array}{l}\text { sample stabilized by addi- } \\
\text { tion of } \mathrm{Y}_{2} \mathrm{O}_{3}\end{array}$ & {$[3]$} \\
$3.1122(7)$ & $4.978(2)$ & $1.600(1)$ & R.T. & & {$[16]$} \\
3.1111 & 4.9788 & 1.6003 & R.T. & & {$[17]$} \\
$3.1115(1)$ & $4.9810(2)$ & $1.6008(1)$ & 299 & & {$[11]$} \\
$3.1112(1)$ & $4.9810(2)$ & $1.6010(1)$ & 291 & & this work
\end{tabular}

\section{Acknowledgments}

This work was partially supported by the grant No. 7 T08A 05319 from the State Committee for Scientific Research (Poland).

\section{References}

[1] G.A. Slack, B.F. Bartram, J. Appl. Phys. 46, 89 (1975).

[2] J.H. Edgar, in: Properties of Group III Nitrides, EMIS Datareviews Series, Ed. J.H. Edgar, British Institution of Electrical Engineers Publ., INSPEC, London 1994, p. 7.

[3] S.N. Ivanov, P.A. Popov, G.V. Egorov, A.A. Sidorov, B.I. Korneev, L.M. Zhukova, B.P. Ryabov, Phys. Solid State 39, 83 (1997) [translated from Fiz. Tverd. Tela 39, 93 (1997)].

[4] S. Burghartz, FZKA Report No. 5616, Forschungszentrum Karlsruhe GmbH, Karlsruhe 1995 .

[5] K. Wang, R.R. Reeber, Mater. Res. Soc. Symp. Proc. 482, 863 (1998).

[6] S. Podsiadło, Thermochim. Acta 256, 367 (1995).

[7] S. Podsiadło, Thermochim. Acta 256, 375 (1995).

[8] J. Thringer, A. Koester, J. Appl. Crystallogr. 26, 135 (1993).

[9] R.A. Young, A. Sakthivel, T.S. Moss, C.O. Paiva-Santos, J. Appl. Crystallogr. 28, 366 (1995)

[10] PDF 46-1212, International Centre for Diffraction Data, PA 1999.

[11] W. Paszkowicz, S. Podsiadło, unpublished. 
[12] G.A. Slack, J. Phys. Chem. Solids 34, 321 (1973).

[13] W.M. Yim, R.J. Paff, J. Appl. Phys. 45, 1456 (1974).

[14] H.F. McMurdie, M.C. Morris, E.H. Evans, B. Paretzkin, J.H. de Groot, C.R. Hubbard, S.J. Carmel, Standard X-ray Diffraction Patterns, NBS Monograph 25 Sec. 12, Institute for Materials Research, National Bureau of Standards, Washington DC 1975, p. 5.

[15] M. Tanaka, S. Nakahata, K. Sogabe, H. Nakata, M. Tobioka, Jpn. J. Appl. Phys. Lett. 36, L1062 (1997).

[16] Y.C. Lan, X.L. Chen, Y.G. Cao, Y.P. Xu, L.D. Xun, T. Xu, J.K. Liang, J. Cryst. Growth 207, 247 (1999).

[17] H. Iwanaga, A. Kunishige, S. Takeuchi, J. Mater. Sci. 35, 2451 (2000). 